\title{
The effect of inhibiting exosomes derived from adipose-derived stem cells via the TGF- $\beta 1 /$ Smad pathway on the fibrosis of keloid fibroblasts
}

\author{
Zhi-Yuan Wu ${ }^{1}$, Hui-Jun Zhang ${ }^{2}$, Zhi-Hong Zhou ${ }^{1}$, Zhan-Peng Li ${ }^{1}$, Si-Mu Liao ${ }^{1}$, Ze-Yong Wu ${ }^{1}$, \\ Hai-Hua Huang, ${ }^{1}$ Yu-Cang Shi ${ }^{1}$ \\ ${ }^{1}$ Department of Plastic Surgery, Affiliated Hospital of Guangdong Medical University, Zhanjiang, China; ${ }^{2}$ Department of Burn Surgery, Affiliated \\ Hospital of Guangdong Medical University, Zhanjiang, China \\ Contributions: (I) Conception and design: YC Shi, ZY Wu; (II) Administrative support: ZY Wu, YC Shi; (III) Provision of study materials or patients: \\ ZH Zhou, ZP Li, SM Liao; (IV) Collection and assembly of data: ZY Wu, HH Huang; (V) Data analysis and interpretation: YC Shi, ZY Wu; (VI) \\ Manuscript writing: All authors; (VII) Final approval of manuscript: All authors. \\ Correspondence to: Yu-Cang Shi. Affiliated Hospital of Guangdong Medical University, No. 57 South of Ren min Avenue, Zhanjiang 524001, China. \\ Email: kelang413@126.com.
}

Background: The main mechanism of keloid formation is that keloid fibroblasts (KFs) apoptosis is inhibited, leading to excessive proliferation. Transforming growth factor- $\beta 1$ (TGF- $\beta 1$ ) is a key signal molecule in the process of regulating cell fibrosis. This paper discusses the effect of adipose-derived stem cell exosomes (ADSCs-EXO) on the proliferation and apoptosis of KFS and its possible mechanism, in order to provide reference for the clinical intervention of hypertrophic scar.

Methods: ADSCs were isolated and cultured from human adipose tissue, the supernatant was collected, and the exosomes secreted by ADSCs-EXO were extracted by ultracentrifugation. At the same time, KFs were cultured from human keloid tissue to P3 generation, and then divided into four groups: control group, experimental group A, experimental group B and experimental group C. KFs were then cultured with four concentrations of $\operatorname{ADSCs-EXO~}(0,1,10$, and $100 \mu \mathrm{g} / \mathrm{mL}$, respectively). After 24 hours, cells in each group were taken to detect the following: proliferation of cells in each group using the cell counting Kit 8 (CCK-8) method, cell migration ability via the Transwell test, cell apoptosis by flow cytometry, collagen synthesis using the hydroxyproline method, messenger ribonucleic acid (mRNA) expression of fibrosis-related genes in each group by real-time fluorescent polymerase chain amplification, and the expression of fibrosis-related proteins in the cells of each group by western blotting.

Results: Compared with the control group, the proliferation rate, migration rate, and collagen synthesis levels in the three experimental groups decreased with the increase of ADSCs-EXO concentration, while the apoptosis rate in the three experimental groups increased with the increase of ADSCs-EXO concentration, and the differences were statistically significant $(\mathrm{P}<0.05)$. Also, compared with the control group, the relative mRNA and protein expression of alpha-smooth muscle actin ( $\alpha$-SMA), TGF- $\beta 1$, and Smad3 in the three groups decreased significantly, while the expression of three kinds of mRNA and protein decreased with the increase of ADSCs-EXO concentration, and the differences were statistically significant $(\mathrm{P}<0.05)$.

Conclusions: ADSCs-EXO may inhibit the proliferation and migration, and promote the apoptosis of KFs by inhibiting the expression of the TGF- $\beta 1 /$ Smad pathway.

Keywords: Transforming growth factor- $\beta$ (TGF- $\beta$ ); adipose stem cells; exosomes; keloid fibroblasts (KFs)

Submitted Dec 03, 2020. Accepted for publication Mar 18, 2021.

doi: $10.21037 / \mathrm{gs}-21-4$

View this article at: http://dx.doi.org/10.21037/gs-21-4 


\section{Introduction}

Keloid is a type of fibroproliferative disorder with excessive fibroblast proliferation, extracellular matrix deposition, and impaired collagen alignment. Keloids is a type of fibroproliferative disease that induced by fibroblasts overgrow and secrete too much collagen, which specifically refers to a kind of pathological scar that protrudes on the skin surface; It is invasive and does not have self-limitation capabilities (1). The causes of keloids are complex, including genetic susceptibility, endocrine factors, infection, local hypertonicity, and excessive inflammation. Among them, endocrine abnormalities, infection, local hypertonicity, and excessive inflammation can further affect the keloids direction and growth speed. Keloids have an obvious family history and a certain genetic susceptibility, which is related to autosomal dominant inheritance and immune factors. The occurrence of keloids can significantly affect the aesthetic appearance, and can even lead to skin dysfunction, which should be well controlled. At present, the treatments for keloids are primarily based on surgical resection, radiation therapy, laser treatment and intradermal drug injection treatment. But keloids specific mechanism is not clear and there is a lack of targeted treatment, therefore, the treatment has limited efficacy and a high recurrence rate. Therefore, discovering efficient and non-destructive intervention methods for hypertrophic scars has always been an important goal of plastic surgeons.

In recent years, stem cells have been widely used in clinical treatment. Adipose-derived stem cells (ADSCs) are a type of mesenchymal stem cell that have a wide range of sources, easy obtainment, and high histocompatibility. Related research shows that ADSCs can promote the highquality healing of tissues, decrease the generation of scars, and reshape the fibrotic matrix, ultimately achieving the effect of reducing fibrosis (2-4). ADSCs-exosomes (ADSCsEXO) are a biologically active substance with repair functions that are isolated from ADSCs. They contain proteins, messenger ribonucleic acid (mRNA) and noncoding RNA. As the medium for transmitting information between ADSCs cells, ADSCs-EXO act to regulate cell proliferation, bone regeneration, and fibroblast collagen synthesis (5).

The mechanism of keloid formation is mainly through the inhibition of apoptosis of keloid fibroblasts (KFs), leading to excessive proliferation of KFs and the generation of a large amount of collagen (6). Transforming growth factor- $\beta$ (TGF- $\beta)$ is a multifunctional factor that regulates cell growth and differentiation. TGF- $\beta 1$ is a key signaling molecule in the regulation of cell fibrosis. Many studies have shown that the presence of TGF- $\beta 1$ in fibrotic diseases can mediate the activation of the TGF- $\beta / \mathrm{Smad}$ signaling pathway (7-9). Smad3 gene and its products can effectively regulate wound healing, and its out-of-control expression may be the real cause of delayed wound healing, non-healing, or excessive scar hyperplasia. The effects of ADSCs-EXO on the proliferation and apoptosis of KFs and its possible mechanism are explored in this study, with a view to providing a reference for clinical keloid intervention. We present the following article in accordance with the MDAR reporting checklist (available at http:// dx.doi.org/10.21037/gs-21-4).

\section{Methods}

\section{Main reagents and instruments}

\section{Main reagents}

Fetal bovine serum and trypsin were purchased from Gibco, USA; Dulbecco's Modified Eagle's medium (DMEM) culture medium and penicillin-streptomycin solution were purchased from Hyclone, USA; I collagenase, phosphatebuffered saline (PBS) buffer, and Annexin V-FITC/ propidium iodide (PI) apoptosis detection kits were purchased from Sigma, USA; anti-TGF- $\beta 1$ antibody, antiSmad3 antibody, anti-Cluster of Differentiation 9 (CD9) antibody, anti-CD63 antibody, anti-Glyceraldehyde3-phosphate dehydrogenase (GAPDH) antibody, and horseradish peroxidase (HRP)-labeled goat anti-rabbit immunoglobulin G ( $\operatorname{IgG}$ ) were purchased from Abcam, USA; Trizol reagent, reverse transcription kit, and realtime polymerase chain reaction (RT-PCR) kit were purchased from TAKARA, Japan; hydroxyproline detection kit was purchased from Nanjing Jiancheng Biotechnology Research Institute, China; bicinchoninic acid (BCA) protein quantitative detection kit, sodium dodecyl sulfate polyacrylamide gel electrophoresis (SDS-PAGE) gel preparation reagent kits, electrochemiluminescence (ECL) kits, and cell counting Kit-8 (CCK-8) kits were purchased from Biyuntian Biotechnology Corporation Limited, China; and Transwell cells were purchased from Corning, USA.

\section{Main instruments}

Low-temperature high-speed centrifuges were purchased from Eppendorf, Germany; ultra-centrifuges were purchased from Beckman, USA; constant temperature 
incubators were purchased from Thermo Scientific, USA; flow cytometers were purchased from BD, USA; inverted phase contrast microscopes were purchased from Olinbath, Japan; fluorescence quantitative PCR instrument was purchased from ABI, USA; transmission electron microscope was purchased from Hitachi, Japan; gel electrophoresis system was purchased from Bio-Rad, USA.

\section{Experimental methods}

\section{ADSC culture in vitro}

ADSCs were obtained from healthy women who underwent liposuction in orthopedic surgery in the hospital [informed consent was signed prior to the operation and this study was approved by the ethics committee of the Affiliated Hospital of Guangdong Medical University (No. PJ2020-021)]. The study was conducted in accordance with the Declaration of Helsinki (as revised in 2013). ADSCs were isolated by trypsin digestion. The specific methodology was as follows. Wash the extracted adipose tissue three times with PBS, add an equal volume of $0.1 \%$ type I collagenase, shake thoroughly in a water bath at $37^{\circ} \mathrm{C}$ for 45 minutes, and add an equal amount of DMEM culture solution containing $10 \%$ fetal bovine serum to stop digestion. Next, centrifuge at 1,200 r/min for 5 minutes at room temperature, take the cell pellet, resuspend the cells with $10 \%$ fetal bovine serum in DMEM, inoculate the cells in a cell culture flask, culture them in a $37{ }^{\circ} \mathrm{C}$ incubator with $5 \%$ carbon dioxide $\left(\mathrm{CO}_{2}\right)$, and replace the culture medium every 3 days. When the cells grow to more than $80 \%$ adherence degree, digest and purify the cells. Plant them in a new culture flask at a ratio of $1: 3$ and subculture to $\mathrm{P} 3$ generation for later use.

\section{$\mathrm{KF}$ cell culture in vitro}

KFs were obtained from 12 keloid patients in the outpatient and inpatient orthopedics department in the hospital (no infection at the diseased site, no local drug injection treatment or radiation treatment were performed, and informed consent was signed prior to the operation and was approved by the ethics committee). Tissue block culture was used for culturing. The keloid skin tissue obtained during surgery was washed 5 to 10 times with PBS, and then sterilized with $1,000 \mathrm{U} / \mathrm{mL}$ penicillin-streptomycin solution for 40 minutes and rinsed with sterile PBS three times. The tissue was subsequently cut into $1 \mathrm{~mm}^{3}$ cells and placed in a cell culture dish. After adherence, DMEM complete medium containing $10 \%$ fetal bovine serum was added and cultured in a $37{ }^{\circ} \mathrm{C}$ incubator with $5 \% \mathrm{CO}_{2}$. The culture medium was changed every 3 days. After the cells grew to more than $80 \%$ adherence degree, they were digested with trypsin and purified, and then planted in a new culture flask at a ratio of $1: 3$ and sub-cultured to $\mathrm{P} 3$ generation for later use.

\section{ADSCs-EXO isolation and identification}

After undergoing ultracentrifugation, the $\mathrm{P} 3$ generation ADSCs cells were cultured in a DMEM culture solution containing $10 \%$ fetal bovine serum in a $37{ }^{\circ} \mathrm{C}$ incubator with $5 \% \mathrm{CO}_{2}$. When the cells were well developed and the density was $1 \times 10^{6}-1 \times 10^{7}$, the cell culture supernatant was collected and the EXO were extracted by ultracentrifugation. The specific method was as follows. Transfer $1 \times 10^{5} \mathrm{~g}$ of the supernatant to a sterile ultracentrifuge tube and centrifuge at $4{ }^{\circ} \mathrm{C}$ for 1 hour. Then, discard the supernatant and add PBS to resuspend the pellet. Next, repeat the centrifugation once, add $200 \mu \mathrm{L}$ of pre-chilled PBS and resuspend to obtain ADSCs-EXO, and store at $-80^{\circ} \mathrm{C}$ for later use. Identification of ADSCsEXO: $10 \mu \mathrm{L}$ of ADSCs-EXO is dropped onto a 300-mesh sample copper net, the excess liquid is wiped off, and the $3 \%$ of phosphotungstic acid solution is dyed for 1 minute. After drying, the morphology of the ADSCs-EXO is observed under a transmission electron microscope. At the same time, western blotting is used to detect the surface markers of the ADSCs-EXO (CD9 and CD63).

\section{Group culture}

P3 generation KF cells were divided into four groups. Cell co-culture experiments were performed with four concentration gradients of $0,1,10$ and $100 \mu \mathrm{g} / \mathrm{mL}$. Experiment A group: DMEM complete medium with $10 \%$ fetal bovine serum $+1 \mu \mathrm{g} / \mathrm{mL}$ ADSCs-EXO; experiment B group: DMEM complete medium with $10 \%$ fetal bovine serum + $10 \mu \mathrm{g} / \mathrm{mL}$ ADSCs-EXO; experimental C group: DMEM complete medium with $10 \%$ fetal bovine serum $+100 \mu \mathrm{g} / \mathrm{mL}$ ADSCs-EXO. KFs were seeded in a 12 -well plate at a density of $2 \times 10^{5}$ cells, and cultured for 24 hours in a $37^{\circ} \mathrm{C}$ incubator with $5 \% \mathrm{CO}_{2}$.

\section{Detection of cell proliferation using the CCK8 method} The specific method was as follows. Collect the cells for 24 hours. Discard the four groups of cells and wash them three times with PBS. Add $100 \mu \mathrm{L}$ of CCK- 8 to each well in a 96-well plate. After cultivation for 2 hours in a $37^{\circ} \mathrm{C}$ incubator with $5 \% \mathrm{CO}_{2}$, the microplate reader was used to determine the optical density (OD) at $450 \mathrm{~nm}$. Each of the 
groups of cells was provided with three duplicate wells, and each well was measured three times to obtain the average.

\section{Detection of apoptosis by flow cytometry}

The specific method was as follows. Collect the cells for 24 hours, add $0.25 \%$ trypsin and digest at $37^{\circ} \mathrm{C}$ for $15 \mathrm{~min}$. Add PBS to prepare suspension, and add into a flow tube, pre-incubate at $6{ }^{\circ} \mathrm{C}$, and suspend in $400 \mu \mathrm{L} 1$ *Annexin $\mathrm{V}$ binding solution cells. Next, add $5 \mu \mathrm{L}$ Annexin V-FITC staining solution, mix and place in a refrigerator at $4{ }^{\circ} \mathrm{C}$ for 15 minutes in the dark, and then add $10 \mu \mathrm{L}$ PI to mix and let stand for 5 minutes. Flow cytometry was used to detect the apoptosis rate $(\%)=($ sum of the number of apoptotic cells in early and late period/total number of cells) $\times 100 \%$.

\section{Detection of migration ability using the Transwell test}

The specific method was as follows. Collect the cells for 24 hours, add serum-free medium, and starve for 12 hours. Subsequently, discard the supernatant, add $0.25 \%$ trypsin and digest at $37^{\circ} \mathrm{C}$ for $15 \mathrm{~min}$. Add PBS to prepare a cell suspension of the same concentration, and add it into a 96-well plate in advance $500 \mu \mathrm{L}$ DMEM culture solution containing $10 \%$ PBS. Next, place the Transwell chamber into the well, add $200 \mu \mathrm{L}$ cell suspension, and place it in an environment of $37{ }^{\circ} \mathrm{C}$ with $5 \% \mathrm{CO}_{2}$ for 72 hours. Then, discard the culture solution, wash it with PBS, $4 \%$ paraformaldehyde was fixed for $15 \mathrm{~min}$, and $0.1 \%$ crystal violet staining solution was stained for 10 minutes. Five fields of view were photographed and recorded under a microscope, and the migrating cells were counted.

\section{Detection of collagen synthesis ability via the hydroxyproline method}

The specific method was as follows. Take $250 \mu \mathrm{L}$ of cell culture solution and standard application solution cultured in $37^{\circ} \mathrm{C}$ for 24 hours, add $0.05 \mathrm{~mL}$ of digestion solution and then place into a $37^{\circ} \mathrm{C}$ water bath for 3 hours. Next, add $0.5 \mathrm{~mL}$ of reagent, then mix and let stand for 10 minutes. Subsequently, add $0.5 \mathrm{~mL}$ of reagent 2 , then mix and let stand for 5 minutes. Finally, add $1 \mathrm{~mL}$ of reagent 3 and place in a $60{ }^{\circ} \mathrm{C}$ water bath for 15 minutes. After centrifugation, take $200 \mu \mathrm{L}$ of the supernatant to measure the absorbance at $550 \mathrm{~nm}$, and calculate the hydroxyproline content using the standard curve.

\section{Detection of relative mRNA expression by RT-PCR}

The specific method was as follows. Total RNA was extracted from four groups of cells using the Trizol method, and RNA concentration was measured by using a ultraviolet (UV) spectrophotometer (Thermo Scientific Evolution 200, USA). Reverse transcription was performed with $1 \mu \mathrm{g}$ of total RNA, and complementary deoxyribonucleic acid (cDNA) was used as a template for real-time fluorescent PCR amplification. After the corresponding cycle threshold (CT) value is read, the relative expression content is calculated using the $2^{-\Delta \Delta \mathrm{Ct}}$ method. The primers were synthesized using bio-organisms, and GAPDH was used as an internal reference.

\section{Detection of relative protein expression by Western blot}

Total proteins from four groups of cells were extracted, and the protein concentration was determined using the BCA method. $10 \%$ polyacrylamide gel electrophoresis and electro transference to polyvinylidene fluoride (PVDF) membrane were then performed. The proteins were blocked with $5 \%$ skim milk, and $5 \mathrm{~mL}$ primary antibody (1:500) was added overnight at $4{ }^{\circ} \mathrm{C}$. HRP-labeled goat anti-rabbit IgG $(1: 5,000)$ was then incubated at $28{ }^{\circ} \mathrm{C}$ for 1 hour. The PVDF film was subsequently developed by electroluminescence, the gel imaging system (Bio-Rad, USA) was used to take pictures, and the Image J software (National Institutes of Health, USA) was used to calculate the gray value.

\section{Observation indicators}

(I) 24-hour appreciation rate of the four groups of cells; (II) 24-hour apoptosis rate of the four groups of cells; (III) 24-hours migration rate of the four groups of cells; (IV) collagen synthesis of four groups of the cells; (V) mRNA and protein expression of $\alpha$-SMA, TGF- $\beta 1, \operatorname{Smad} 3$ in the four groups of cells.

\section{Statistical analysis}

The SPSS 21.0 (International Business Machines Corporation, USA) was used for statistical analysis of the data. The measurement data was expressed in the form of $\bar{x} \pm$ s. The comparison of the measurement data between the four groups was analyzed by single factor analysis of variance. The post hoc comparison was performed using Least-Significant Difference (LSD)-t test. The difference was statistically significant at $\mathrm{P}<0.05$. 

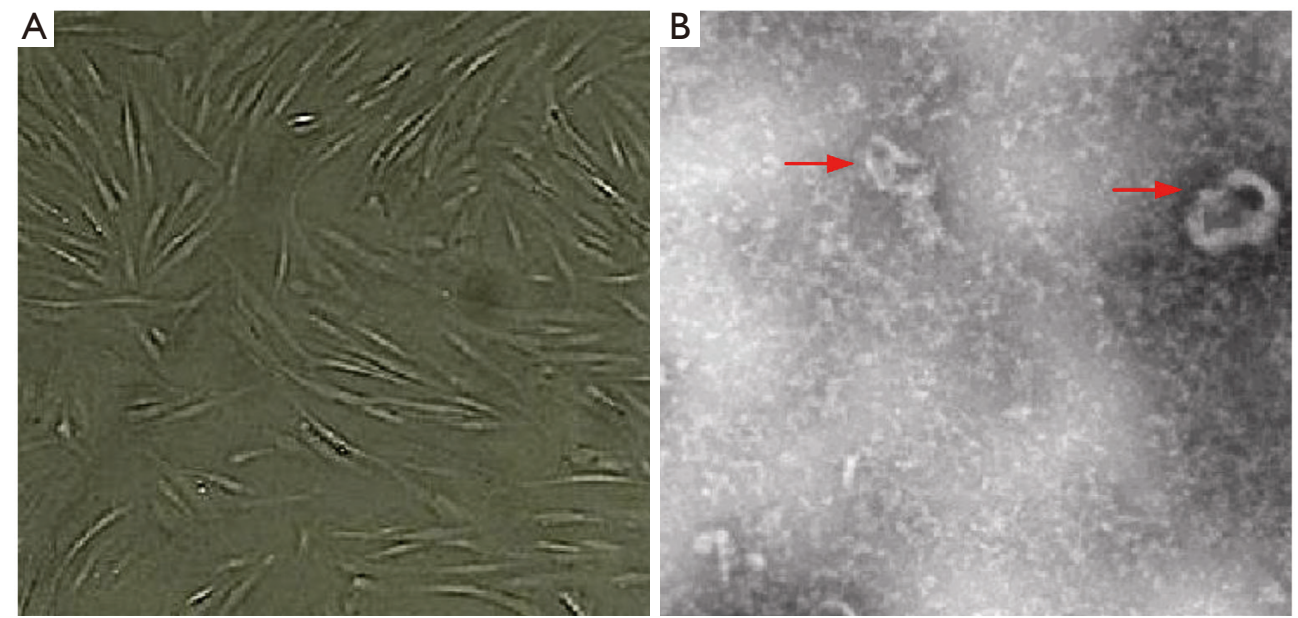

Figure 1 Isolation and identification of ADSCs and EXO (phosphotungstic acid solution staining): (A) ADSCs P3 cell morphology under phase contrast microscope (50x); (B) ADSCs-EXO morphology under transmission electron microscope (5,000x). The red arrows indicate ADSCs-EXO. ADSC, adipose-derived stem cell.

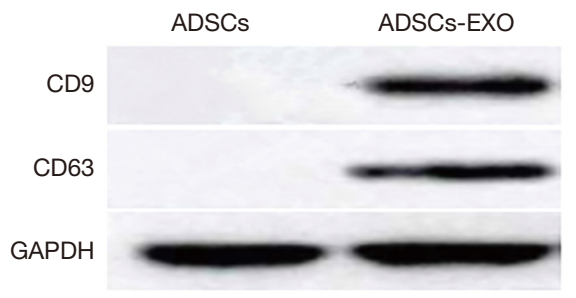

Figure 2 Protein expression of ADSCs-EXO surface specific markers CD9 and CD63. ADSC, adipose-derived stem cell.

\section{Results}

\section{Isolation and identification of ADSCs-EXOs}

The P3 generation ADSCs cells were long and spindleshaped, and were arranged in a vortex (observed by microscope). The cytoplasm was transparent and the outline of the cells was clear, as shown in Figure 1A. ADSCs-EXO were round/oval-shaped vesicle structures, clustered or scattered, with a diameter of about $50-150 \mathrm{~nm}$ (observed by transmission electron microscope), as shown in Figure $1 B$.

The expression levels of ADSCs-EXO surface specific markers (CD9 and CD63) proteins were significantly higher than those of the ADSCs cells, which indicated that the ADSCs-EXO were successfully extracted (Figure 2).

\section{Cell proliferation}

After 24 hours of KFs culture with ADSCs-EXO at different concentrations, the cell proliferation in the control group was used as the contrast (100\%). The cell proliferation in experimental group A was $(38.55 \pm 1.95) \%$, experimental group B was $(56.91 \pm 1.37) \%$, and experimental group $\mathrm{C}$ was $(38.55 \pm 1.95) \%$. Compared with the control group, the cell proliferation rate of the three experimental groups was reduced to a certain extent. This rate decreased as the concentration of ADSCs-EXO increased, and the differences were statistically significant $(\mathrm{P}<0.05$, Figure 3$)$.

\section{Cell apoptosis}

After the cultivation of KFs and ADSCs-EXO at different concentrations for 24 hours, the apoptosis rate was $(8.11 \pm 0.05) \%$ in the control group, $(23.52 \pm 1.53) \%$ in experimental group A, $(23.52 \pm 1.53) \%$ in experimental group B, and $(39.47 \pm 1.82) \%$ in experimental group C. Compared with the control group, the apoptosis rate of the three experimental groups increased to a certain extent. This rate increased as the concentration of ADSCs-EXO increased, and the differences were statistically significant $(\mathrm{P}<0.05$, Figures 4,5$)$.

\section{Cell migration}

After cultivation of KFs and ADSCs-EXO at different concentrations for 24 hours, the cell migration rate was $(100 \pm 0.75) \%$ in the control group, $(79.06 \pm 1.82) \%$ in experimental group A, $(56.31 \pm 1.72) \%$ in experimental 


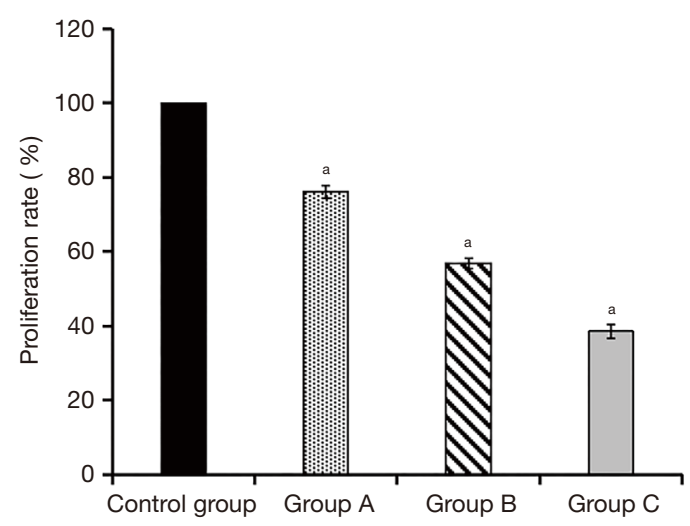

Figure 3 Comparison of 24 h cell proliferation rates of the four groups of cells. ${ }^{\text {a }}$, the standard deviation of each set of data.

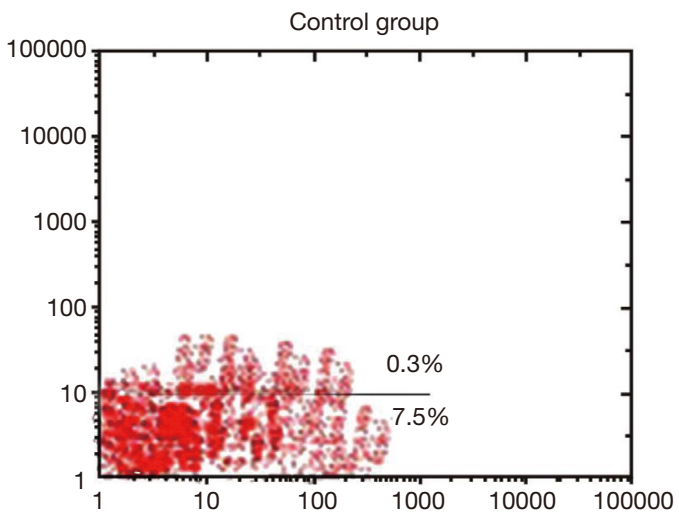

Group B

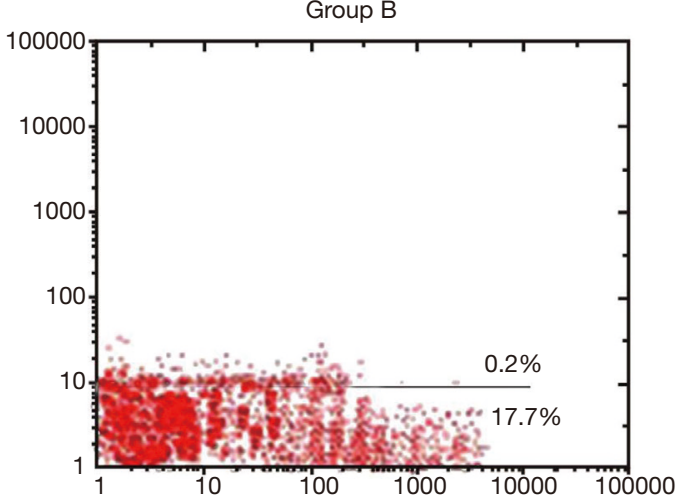

Figure 4 Detection of apoptosis by flow cytometry. group B, and $(36.73 \pm 1.17) \%$ in experimental group C. Compared with the control group, the cell migration rate of the three experimental groups decreased to a certain extent. This rate decreased as the concentration of ADSCs-EXO increased, and the differences were statistically significant $(\mathrm{P}<0.05$, Figures 6, 7).

\section{Cellular collagen synthesis}

After cultivation of KFs and ADSCs-EXO at different concentrations for 24 hours, the collagen synthesis level was $(3.76 \pm 0.45) \mathrm{mg} / \mathrm{L}$ in the control group, $(2.19 \pm 0.12) \mathrm{mg} / \mathrm{L}$ in experimental group $\mathrm{A},(1.63 \pm 0.27) \mathrm{mg} / \mathrm{L}$ in experimental group $\mathrm{B}$, and $(0.97 \pm 0.11) \mathrm{mg} / \mathrm{L}$ in experimental group $\mathrm{C}$.
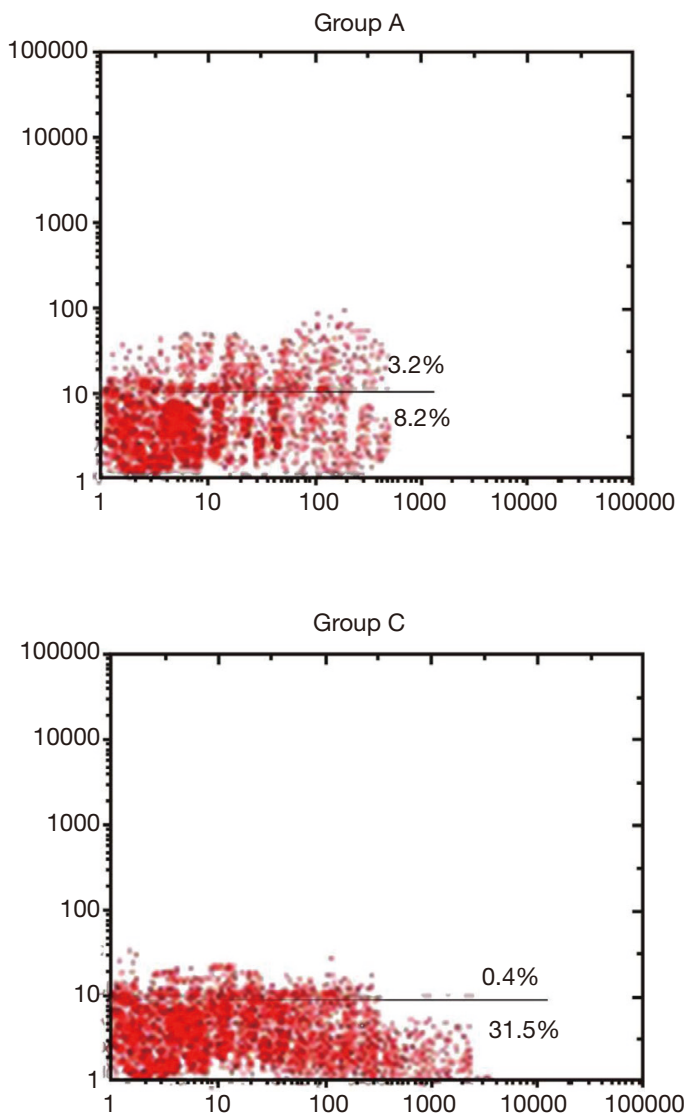


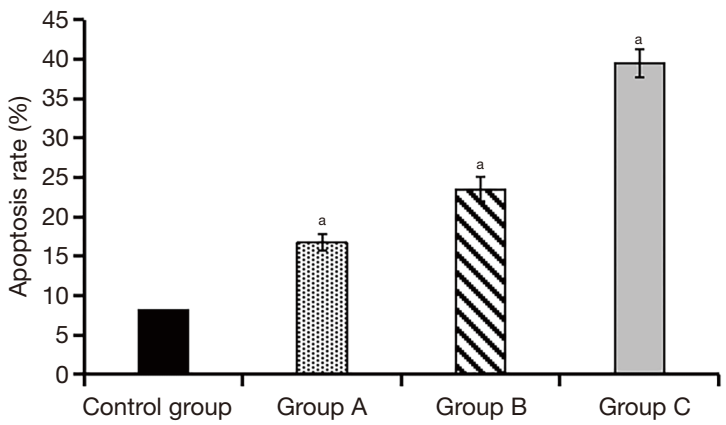

Figure 5 Comparison of apoptotic rates at 24 h between the four groups of cells.
Compared with the control group, the collagen synthesis level of the three experimental groups decreased to a certain extent. This decrease was more significant as the concentration of ADSCs-EXO increased, and the differences were statistically significant $(\mathrm{P}<0.05$, Figure 8$)$.

\section{$m R N A$ expression of $\alpha-S M A, T G F-\beta 1$, and Smad 3 in the cells}

Compared with the control group, the relative mRNA expression levels of $\alpha$-SMA, TGF- $\beta 1$, and Smad3 in the
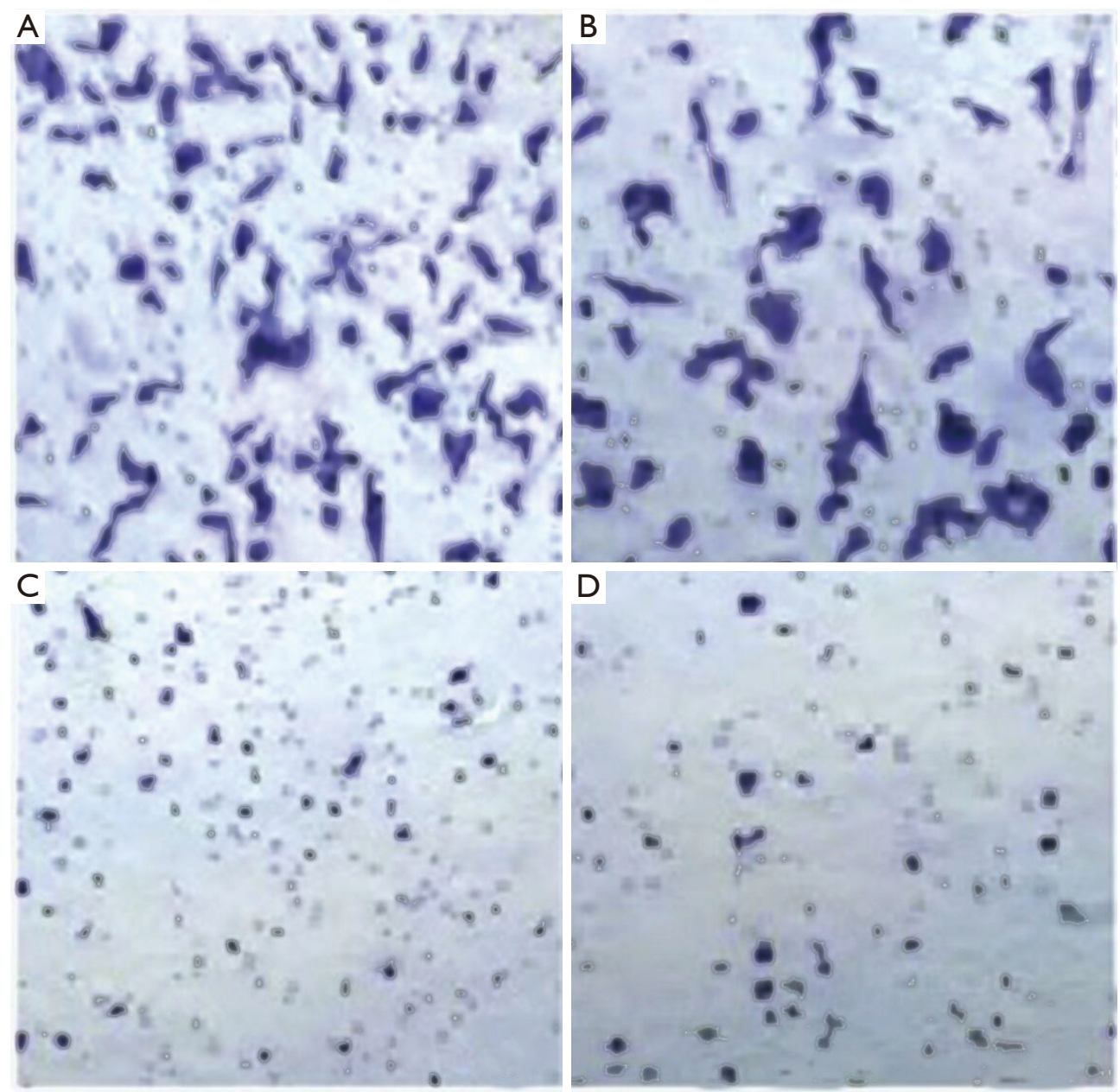

Figure 6 Cell migration in four groups (dyeing with crystal violet, $\times 100$ ): (A) control group; (B) experimental group A; (C) experimental group B; (D) experimental group C. 


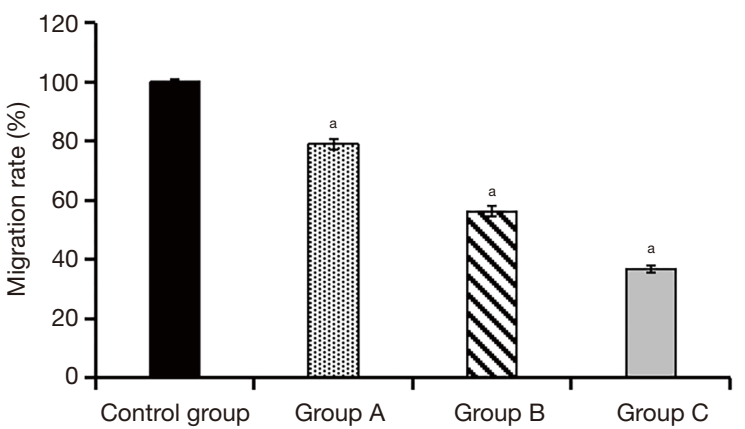

Figure 7 Comparison of cell migration between the four groups. a, compared with the control group, $\mathrm{P}<0.05$.

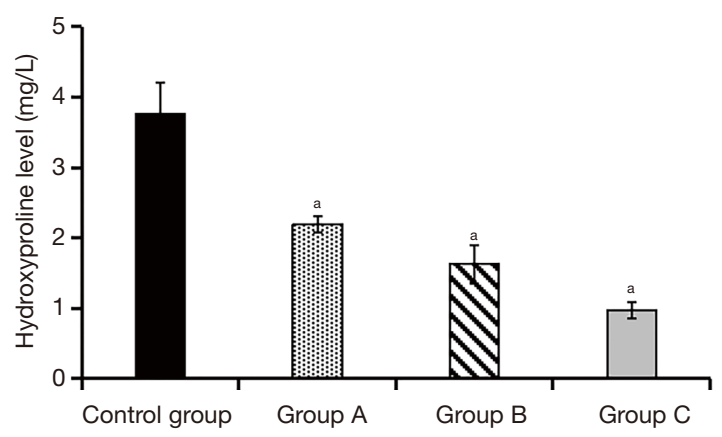

Figure 8 Comparison of collagen synthesis in the four groups of cells. a, compared with the control group, $\mathrm{P}<0.05$.

three experimental groups were significantly reduced. As the ADSCs-EXO concentration increased, the expression levels of the three mRNAs decreased. The expression levels in the experimental group $\mathrm{C}$ were the lowest, and the differences were statistically significant $(\mathrm{P}<0.05$, Table 1).

\section{Proteins expression of $\alpha-S M A, T G F-\beta 1$, and Smad 3 in the cells}

Compared with the control group, the relative expression of $\alpha$-SMA, TGF- $\beta 1$, and Smad3 proteins in the three experimental groups decreased to a certain extent. As the concentration of ADSCs-EXO increased, the expression levels of the three proteins were lower. The expression levels in experimental group $\mathrm{C}$ were the lowest, and the differences were statistically significant $(\mathrm{P}<0.05$, Table 2, Figure 9).

\section{Discussion}

After the skin is damaged, it will undergo a series of pathophysiological processes including inflammation, proliferation, and remodeling. The first stage of wound healing is the inflammation stage. The inflammatory and immune responses are activated after tissue damage, which will generally last for approximately 2-3 days. In addition, the coagulation cascade reaction, complement activation, and platelet degranulation are occurring simultaneously. The second stage is proliferation, which is primarily characterized by the formation of new tissues, and may last 3-6 weeks. Keratinocytes are formed and migrate to damaged tissues, and new blood vessels are also formed. In the late proliferation stage, fibroblasts and myofibroblasts produce extracellular matrix (mainly collagen), and this accumulated collagen forms most of the scar. When the wound is closed, it enters the third stage; the remodeling stage. At this time, type III collagen in the excessive extracellular matrix will be degraded and converted into mature type I collagen, which will last for at least 1 year (10). These three stages include a complex regulatory network, and an imbalance in any of these stages will lead to abnormal wound healing and the formation of pathological scars.

ADSCs, like other stem cells, have the potential for multi-directional differentiation and self-renewal. The discovery of their role in tissue regeneration and repair offers a positive outlook for the treatment of fibrotic diseases. Many studies have confirmed that ADSCs can reduce tissue fibrosis, and have certain application values in liver fibrosis, pulmonary fibrosis, and myocardial fibrosis (11-13). However, there are few studies on the efficacy of ADSCs in skin fibrosis, and the research on its application mechanism in pathological scars remains incomplete. At present, there is no clear conclusion about the effectiveness of ADSCs in the treatment of keloids (14).

In this study, ADSCs were initially isolated and cultured by collagenase digestion. ADSCs were observed (under phase contrast microscope) as long spindles arranged in a swirling pattern. ADSCs-EXO were then extracted by ultracentrifugation. ADSCs-EXO are extracellular vesicles with a uniform shape and size. They were round/oval in shape (observed with transmission electron microscope) and had a diameter of approximately $50-150 \mathrm{~nm}$. ADSCsEXO surface-specific marker proteins (CD9 and CD63) were expressed and found to be positive, indicating that the ADSCs-EXO were successfully isolated.

KFs were co-cultured with ADSCs-EXO at different concentrations for 24 hours. The results showed that the cell proliferation rate, migration rate, and collagen synthesis levels of the three groups were reduced compared 
Table 1 Comparison of the relative mRNA expression levels of $\alpha$-SMA, TGF- $\beta 1$, and Smad 3 in the four groups of cells

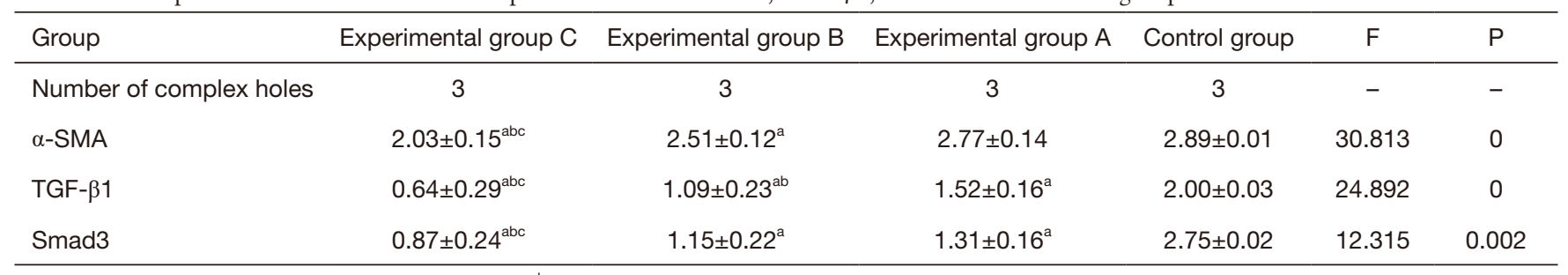

${ }^{a}$, compared with the control group, $\mathrm{P}<0.05 ;{ }^{b}$, compared with experimental group $\mathrm{A}, \mathrm{P}<0.05 ;{ }^{\mathrm{c}}$, compared with experimental group $\mathrm{B}$, $\mathrm{P}<0.05$.

Table 2 Comparison of relative protein expression levels of $\alpha$-SMA, TGF- $\beta 1$, and Smad 3 in the four groups of cells

\begin{tabular}{|c|c|c|c|c|c|c|}
\hline Group & Experiment group C & Experiment group B & Experiment group A & Control group & $\mathrm{F}$ & $\mathrm{P}$ \\
\hline$\alpha$-SMA & $0.97 \pm 0.20^{\mathrm{abc}}$ & $1.82 \pm 0.13^{\mathrm{ab}}$ & $2.17 \pm 0.09^{a}$ & $2.43 \pm 0.02$ & 74.255 & 0 \\
\hline TGF- $\beta 1$ & $0.71 \pm 0.22^{\mathrm{abc}}$ & $1.11 \pm 0.15^{\mathrm{ab}}$ & $1.54 \pm 0.12^{\mathrm{a}}$ & $2.00 \pm 0.04$ & 42.596 & 0 \\
\hline Smad3 & $1.30 \pm 0.29^{\mathrm{abc}}$ & $1.75 \pm 0.26^{\mathrm{ab}}$ & $2.27 \pm 0.16^{a}$ & $3.00 \pm 0.03$ & 36.693 & 0 \\
\hline
\end{tabular}

${ }^{a}$, compared with the control group, $\mathrm{P}<0.05 ;{ }^{b}$, compared with experimental group $\mathrm{A}, \mathrm{P}<0.05 ;{ }^{\mathrm{c}}$, compared with experimental group $\mathrm{B}$, $\mathrm{P}<0.05$.

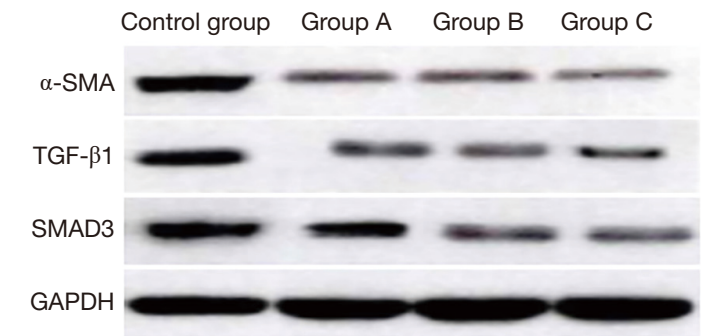

Figure 9 Western blot detection of $\alpha$-SMA, TGF- $\beta 1$, and Smad3 protein expression in the four groups of cells. ADSCs-EXO may inhibit the proliferation, migration, and collagen synthesis of KFs by inhibiting the expression of TGF- $\beta 1 /$ Smad pathway, and promote the apoptosis of KFs, thus reducing scar formation. In the future, ADSCs-EXO is expected to be one of the means of scar intervention.

to the control group without ADSCs-EXO. Furthermore, these were found to decrease with the increased concentration of ADSCs-EXO. The apoptosis rate of the three experimental groups increased to a certain extent. This rate increased as the concentration of ADSCs-EXO increased. Keloids are composed of type I and type III collagen bundles that are abnormally thickened, irregularly branched, and disorderly arranged. They exhibit strong collagen synthesis, increased proportions of type III collagen, and a disordered proliferation stage, which leads to excessive collagen deposition (15). Wang et al. pointed out that ADSCs-EXO can reduce keloid formation, and that the Matrix Metalloproteinase 3 (MMP3) protein level is regulated through the Mitogen-Activated Protein Kinases/Extracellular Regulated Kinase (ERK/MAPK) pathway thereby regulating extracellular mechanisms to complete remodeling (16). Xiang et al. co-cultured KFs cells with different proportions of ADSCs, and discovered that ADSCs can significantly inhibit the proliferation, migration, and collagen synthesis of KFs. This inhibitory effect is significantly enhanced with the increased proportion of ADSCs (17). However, Hu et al. adopted plasma-derived EXO to subcutaneously inject mouse fullthickness skin wounds, and found that plasma-derived EXO can promote fibroblast proliferation and migration, and accelerate epithelial regeneration and wound healing (18). $\mathrm{Hu}$ et al. also demonstrated that ASCs-EXO can be absorbed by fibroblasts, stimulate cell proliferation, migration, and collagen synthesis, and thereby promote skin wound healing (19). Additionally, it has been shown that ASCs-EXO can play different roles in different stages of wound healing. They can promote fibroblast proliferation in the early stage of wound healing, and thus promote wound closure. However, in the late stage of wound healing, remodelling of the extracellular matrix can 
reduce keloid tissue when the fibroblasts proliferate into keloids excessively.

ADSCs-EXO can also modify and activate target cells through paracrine or endocrine signaling, thereby regulating the physiological activities of target cells (20). Compared with the control group, the relative mRNA and protein expression levels of $\alpha$-SMA, TGF- $\beta 1$, and Smad3 in the three experimental groups were significantly reduced, and the expression levels of the three mRNAs and proteins decreased with the increasing concentration of ADSCs-EXOs. TGF- $\beta 1$ imbalance is one of the key drivers of keloid formation. Its overexpression causes downstream Smad and other signals to be activated, which in turn promotes fibroblast proliferation and secretes more extracellular matrix. Zhai et al. measured the expression of TGF- $\beta 1$ in pathological KFs, and found that the mRNA and protein expression of TGF- $\beta 1$ was significantly higher compared to normal skin tissues (21). Li et al. adopted ADSC-conditioned medium to culture keloid tissue, and noted that that it can effectively reduce the expression of collagen and $\alpha$-SMA (22). Chu et al. used ADSCs to transplant into the hypertrophic scar tissue of rabbit ears, and reported that ADSCs can inhibit the mRNA and protein expression of $\alpha$-SMA and reduce the content of collagen I in fibroblasts (23). Fang et al. confirmed that micro RNAs (miRNAs) in umbilical cord-derived mesenchymal stem cell EXO can express the TGF- $\beta$ / SMAD2 pathway, thereby inhibiting $\alpha$-SMA expression and reducing collagen deposition, which may be useful in preventing scarring during wound healing (24).

In contrast to the research ideas proposed in this study, a literature review by Ogawa pointed out that scars are a kind of benign hyperplasia, not tumors. Thus, treatments should target blood vessels and endothelial cells as opposed to fibroblasts. Keloid treatment should focus on preventing and reducing the inflammation reaction (25). Therefore, it is necessary for future studies to comprehensively explore effective means of scar intervention by integrating fibrosis and inflammation response regulation mechanism.

In conclusion, ADSCs-EXO may inhibit the expression of the TGF- $\beta 1 /$ Smad pathway, and thereby inhibit the proliferation, migration, and collagen synthesis of KFs. The apoptosis of KFs is also improved, thus reducing the keloid. Moving forward, it is expected that ADSCs-EXO will be used as one of the means for keloid treatment.

\section{Acknowledgments}

Funding: This research was funded by the Science and Technology Planning Projects of Zhanjiang City in Guangdong Province (2016A01021).

\section{Footnote}

Reporting Checklist: The authors have completed the MDAR reporting checklist. Available at http://dx.doi.org/10.21037/ gs-21-4

Data Sharing Statement: Available at http://dx.doi. org/10.21037/gs-21-4

Conflicts of Interest: All authors have completed the ICMJE uniform disclosure form (available at http://dx.doi. org/10.21037/gs-21-4). The authors have no conflicts of interest to declare.

Ethical Statement: The authors are accountable for all aspects of the work in ensuring that questions related to the accuracy or integrity of any part of the work are appropriately investigated and resolved. This study was approved by the medical ethics committee of the Affiliated Hospital of Guangdong Medical University (No. PJ2020-021). Informed consent was signed prior to the operation. The study was conducted in accordance with the Declaration of Helsinki (as revised in 2013).

Open Access Statement: This is an Open Access article distributed in accordance with the Creative Commons Attribution-NonCommercial-NoDerivs 4.0 International License (CC BY-NC-ND 4.0), which permits the noncommercial replication and distribution of the article with the strict proviso that no changes or edits are made and the original work is properly cited (including links to both the formal publication through the relevant DOI and the license). See: https://creativecommons.org/licenses/by-nc-nd/4.0/.

\section{References}

1. Moon JH, Kwak SS, Park G, et al. Isolation and characterization of multipotent human keloidderived mesenchymal-like stem cells. Stem Cells Dev 2008;17:713-24. 
2. Yoshimura K, Sato K, Aoi N, et al. Cell-assisted lipotransfer for facial lipoatrophy: efficacy of clinical use of adipose-derived stem cells. Dermatol Surg 2008;34:1178-85.

3. Qu M, Song N, Chai G, et al. Pathological niche environment transforms dermal stem cells to keloid stem cells: a hypothesis of keloid formation and development. Med Hypotheses 2013;81:807-12.

4. Mizuno H. Adipose-derived stem cells for tissue repair and regeneration: ten years of research and a literature review. J Nippon Med Sch 2009;76:56-66.

5. Kim HS, Choi DY, Yun SJ, et al. Proteomic analysis of microvesicles derived from human mesenchymal stem cells. J Proteome Res 2012;11:839-49.

6. Lee G, Hunter-Smith DJ, Rozen WM. Autologous fat grafting in keloids and hypertrophic scars: a review. Scars Burn Heal 2017;3:2059513117700157.

7. Akiko H, Ye GC. TGF- $\beta$ Signaling From Receptors to Smads. Cold Spring Harb Perspect Biol 2016;8(9).

8. Meng XM, Tang PM, Li J, et al. TGF- $\beta /$ Smad Signaling in Renal Fibrosis. Front Physiol 2015;6:82.

9. Xu F, Liu C, Zhou D, et al. TGF- $\beta$ /SMAD Pathway and Its Regulation in Hepatic Fibrosis. J Histochem Cytochem 2016;64:157-67.

10. Lee HJ, Jang YJ. Recent Understandings of Biology, Prophylaxis and Treatment Strategies for Hypertrophic Scars and Keloids. Int J Mol Sci 2018;19:711.

11. Faner R, Rojas M, MacNee W, et al. Abnormal lung aging in chronic obstructive pulmonary disease and idiopathic pulmonary fibrosis. Am J Respir Crit Care Med 2012;186:306-13.

12. Qu Y, Zhang QD, Cai XB, et al. Exosomes Derived From miR-181-5p-modified Adipose-Derived Mesenchymal Stem Cells Prevent Liver Fibrosis via Autophagy Activation. J Cell Mol Med 2017;21:2491-502.

13. Nazhvani FD, Haghani I, Nazhvani SD, et al. Regenerative effect of mesenteric fat stem cells on ccl4induced liver cirrhosis, an experimental study. Ann Med Surg (Lond) 2020;60:135-9.

14. Stępniewski J, Tomczyk M, Andrysiak K, et al. Human Induced Pluripotent Stem Cell-Derived Cardiomyocytes, in Contrast to Adipose Tissue-Derived Stromal Cells, Efficiently Improve Heart Function in Murine Model of Myocardial Infarction. Biomedicines 2020;8:578.

15. Rabello FB, Souza CD, Farina Júnior JA. Update on hypertrophic scar treatment. Review Clinics (Sao Paulo) 2014;69:565-73.

16. Wang L, Hu L, Zhou X, et al. Exosomes Secreted by
Human Adipose Mesenchymal Stem Cells Promote Scarless Cutaneous Repair by Regulating Extracellular Matrix Remodelling. Sci Rep 2017;7:13321.

17. Xiang L, Zhixian W, Hong WL, et al. Molecular mechanism underlying the effect of adipose-derived stem cells on the proliferation of keloid fibroblasts. J Tissue Eng 2019;23:61.

18. Hu Y, Rao SS, Wang ZX, et al. Exosomes From Human Umbilical Cord Blood Accelerate Cutaneous Wound Healing Through miR-21-3p-mediated Promotion of Angiogenesis and Fibroblast Function. Theranostics 2018;8:169-84.

19. Hu L, Wang J, Zhou X, et al. Exosomes Derived From Human Adipose Mensenchymal Stem Cells Accelerates Cutaneous Wound Healing via Optimizing the Characteristics of Fibroblasts. Sci Rep 2016;6:32993.

20. Huang SP, Huang CH, Shyu JF, et al. Promotion of wound healing using adipose-derived stem cells in radiation ulcer of a rat model. J Biomed Sci 2013;20:51.

21. Zhai XX, Tang ZM, Ding JC, et al. Expression of TGFssimTOR Signaling Pathway in Pathological Scar Fibroblasts. Mol Med Rep 2017;15:3467-72.

22. Li Y, Zhang W, Gao JX, et al. Adipose Tissue-Derived Stem Cells Suppress Hypertrophic Scar Fibrosis via the p38/ MAPK Signaling Pathway. Stem Cell Res Ther 2016;7:102.

23. Chu H, Wang Y, Wang X, et al. Effects of Transplanted Adipose Derived Stem Cells on the Expressions of $\alpha$-SMA and DCN in Fibroblasts of Hypertrophic Scar Tissues in Rabbit Ears. Exp Ther Med 2018;16:1729-34.

24. Fang S, Xu C, Zhang YT, et al. Umbilical CordDerived Mesenchymal Stem Cell-Derived Exosomal MicroRNAs Suppress Myofibroblast Differentiation by Inhibiting the Transforming Growth Factor-oblasts Fibroblast Functionnd Healing. Stem Cells Transl Med 2016;5:1425-39.

25. Ogawa R. Keloid and Hypertrophic Scars Are the Result of Chronic Inflammation in the Reticular Dermis. Int J Mol Sci 2017;18:606.

(English Language Editor: A. Kassem)

Cite this article as: $\mathrm{Wu} \mathrm{ZY,} \mathrm{Zhang} \mathrm{HJ,} \mathrm{Zhou} \mathrm{ZH,} \mathrm{Li} \mathrm{ZP,}$ Liao SM, Wu ZY, Huang HH, Shi YC. The effect of inhibiting exosomes derived from adipose-derived stem cells via the TGF- $\beta 1 /$ Smad pathway on the fibrosis of keloid fibroblasts. Gland Surg 2021;10(3):1046-1056. doi: 10.21037/gs-21-4 